\title{
Frontiers in imaging magnetism with polarized x-rays
}

\author{
Peter Fischer ${ }^{1,2 *}$ \\ Center for X-ray Optics, Materials Sciences Division, Lawrence Berkeley National Laboratory, Berkeley, CA, USA \\ 2 Physics Department, University of California, Santa Cruz, Santa Cruz, CA, USA
}

\section{Edited by:}

Christopher Marrows, University of Leeds, UK

Reviewed by:

Sean Langridge, Science and Technology Facilities Council, UK Dario Alejandro Arena, Brookhaven

National Laboratory, USA

*Correspondence:

Peter Fischer, Center for X-ray

Optics, Materials Sciences Division,

Lawrence Berkeley National

Laboratory, MS 2-400, 1 Cyclotron

Rd, Berkeley, CA 94720, USA

e-mail:pifischer@Ibl.gov
Although magnetic imaging with polarized $x$-rays is a rather young scientific discipline, the various types of established $x$-ray microscopes have already taken an important role in state-of-the-art characterization of the properties and behavior of spin textures in advanced materials. The opportunities ahead will be to obtain in a unique way indispensable multidimensional information of the structure, dynamics and composition of scientifically interesting and technologically relevant magnetic materials.

Keywords: X-ray spectromicroscopy, Fresnel zone plates, spin dynamics, nanomagnetism, x-ray magnetic circular dichroism
Although magnetism is among the oldest physical phenomena known to mankind, the magnetic properties of condensed matter continue to be among the most exciting scientific topics in solid state physics. Magnetism is the backbone of numerous current and future technologies having penetrated our daily life. Applications involving magnetic materials range from nanoscale devices in spintronics [1] that have revolutionized information and sensor technologies to large permanent magnets [2] critical to power generation, transmission and conversion and transportation, e.g., with electric vehicles. Despite this massive technological use of magnetic materials, a fundamental understanding of magnetism, which would allow predicting e.g., a lightweight, sustainable and high performing material for permanent magnets, is still missing.

From the scientific point of view, it is the spin of the electron and the coupling and competing interactions of all spins in a system, which constitutes the fundamental theoretical concept to describe magnetism and magnetic behavior. The exchange interaction is the strongest interaction and favors either parallel or antiparallel alignment of the spins thus giving rise to ferroor antiferromagnetism. The spin-orbit interaction is currently a topic of paramount current interest to the magnetism community, specifically as novel effects occur, when e.g., $3 \mathrm{~d}$ transition metals such as $\mathrm{Fe}, \mathrm{Co}, \mathrm{Ni}$ are interfaced with high $\mathrm{Z}$ materials, e.g., Pt, Ta, thus adding a large spin-orbit coupling. Chiral interactions, such as the Dzyaloshinkii-Moriya interaction, favor non-collinear spin arrangements, and introduces e.g., a certain handedness, which not only gives rise to topologically protected spin textures such as skyrmions, but impacts also, e.g., domain wall motion in nanowires [3].

The magnetic anisotropy prefers certain orientation of the spins and therefore ultimately enables concepts e.g., to store magnetic information. One of the problems in magnetic recording is that higher anisotropy magnetic materials are needed to increase thermal stability. On the other hand, a higher anisotropy requires increased magnetic fields for magnetization reversal, which has engineering limitations. The most promising path to bypass this trilemma is heat assisted magnetic recording [4], where temporarily the anisotropy is locally reduced by heating thus allowing for switching locally the magnetization with much lower magnetic fields. The major challenge is to combine two disparate technologies, namely solid state magnetism with optics and thermodynamics. However, these efforts might bear even more opportunities, than just enabling a higher storage density. The fastest way to switch magnetization was shown to be achievable with single fsec short laser pulses. Following the stunning observation by Beaurepaire [5], that the demagnetization of $\mathrm{Ni}$ can happen on a few fsec time scale, the phenomenon of alloptical magnetic switching (AOS) has emerged since then as one of the most vibrant research areas in magnetism [6]. Whereas in the beginning, this effect was mostly observed in exotic magnetic materials, such as GdFe, very recently, it was shown that AOS can also occur in $\mathrm{FePt}$ [7], which is one of the dominant materials in magnetic storage technologies. This means, that writing magnetic information a million times faster than what can be done today might come into reach in a foreseeable future.

Beyond the time scales of magnetism, specifically how one can control the dynamics of spin systems, e.g., by application of external magnetic and electric fields, or through electronic or photon pulses a basic understanding of the fundamental magnetic length scales, specifically how spins arrange e.g., in magnetic domains taking into account the competition of those various interactions seems to be another key to gain fundamental insight into magnetism.

Imaging magnetic textures is very appealing and a large variety of magnetic microscopy techniques have been developed [8] The ultimate goal is to provide spatial resolution down to fundamental magnetic length scales, i.e., below $10 \mathrm{~nm}$, a temporal resolution down to the fsec regime with "snapshot" capability and to obtain quantitative and element-specific magnetic information with almost single spin sensitivity. Both, fundamental magnetic length and time scales depend on magnetic materials properties, 
such as anisotropy and exchange interactions and as such vary with the magnetic system under investigation.

Magnetic microscopies using polarized X-rays are among the most promising candidates to achieve those goals. Their development to achieve ultimate performances has therefore seen a tremendous impetus over the last two decades [9]. The underlying fundamental physical processes enabling magnetic imaging with $\mathrm{x}$-rays are $\mathrm{x}$-ray dichroism effects, which are used as strong contrast mechanisms and which can be seen as the $\mathrm{x}$-ray counterpart to Kerr and Faraday effects that are well-known from optics. Different to magneto-optics, x-ray dichroism effects allow determining in a quantitative way local magnetic moments [10]. Specifically, $\mathrm{x}$-ray magnetic circular dichroism probes the projection of the magnetization onto the photon propagation direction and $\mathrm{x}$-ray magnetic linear dichroism is sensitive to the square of the magnetization. The former is therefore well suited for collinear (ferro- and ferrimagnetism), and non-collinear (spin chiral) structures, whereas the latter can be used to image antiferromagnetic spin textures. With the wavelength of soft x-rays in the $\mathrm{nm}$ regime and those of hard $\mathrm{x}$-rays even extending down into the sub-nm regime, a diffraction limited spatial resolution for any $\mathrm{x}$-ray microscopy is set well into the scientifically interesting and technologically relevant nano- and sub-nanometer regime for magnetic materials.

Sources of polarized $\mathrm{x}$-rays have become increasingly powerful over the last two decades specifically in terms of available photon flux and even more with regard to peak flux. Specifically, at X-ray Free Electron Lasers (XFEL), the peak intensity of photons outnumbers other probes for magnetic studies, e.g., neutrons by many orders of magnitudes, which allows x-rays to address even small magnetic cross sections, tiny fraction of materials or to probe with very high sensitivity. As the $\mathrm{x}$-rays occur in short $\mathrm{x}$-ray bursts, these $\mathrm{x}$-ray flashes can be used to study the dynamics of spin systems extending from the sub-ns down to the fsec regime [11]. At the same time, lab based x-ray sources originating from high harmonic generation plasma sources are starting to extend into the $\mathrm{x}$-ray regime with fsec (or even asec) time structures [12]. Complementary to XFELs are current developments and concepts to build diffraction limited storage rings [13], which could offer the opportunity to utilize x-ray vortex beams carrying high angular momentum [14]. It can be anticipated, that these future sources will open completely new windows to look at magnetic materials.

About 25 years ago, magnetic x-ray spectroscopy started with pioneering experiments at the Fe K-edge at second generation synchrotrons, which at that time were dominated and controlled by high energy physics research. Due to the missing spin-orbit interaction for s-electrons, which is the initial state at the $\mathrm{Kx}$-ray absorption edge, X-ray Magnetic Circular Dichroism (XMCD) at $\mathrm{K}$-edges is minute and was therefore considered in the beginning a niche application only. This situation changed drastically with the discovery of large (up to tens of percent) XMCD effects at the L edges in $3 \mathrm{~d}$ transition metals [15] and more or less at the same time the theoretical development of magneto-optical sum rules $[16,17]$, which enabled XMCD spectroscopy to become a quantifiable method capable to determine local magnetic spin and orbital moments separately [18]. The strong $\mathrm{x}$-ray dichroism effects, which also occur in $4 \mathrm{~d}, 4 \mathrm{f}$, and 5 f systems, suggested using $\mathrm{XMCD}$ as contrast mechanism in imaging magnetic materials. Soon after, both the surface sensitive $\mathrm{X}$-ray photoemission electron microscopy (X-PEEM) [19] and the bulk sensitive full field transmission soft $\mathrm{X}$-ray microscopy (TXM) [20] using diffractive Fresnel zone plate (FZP) optics demonstrated their use to image magnetic domains at high spatial resolution. Scanning transmission $\mathrm{x}$-ray microscopies (STXM), which utilize the focusing properties of $\mathrm{x}$-ray optics to illuminate a nanoscale spot on the sample entered the field, once an engineering solution was established to handle the mechanical instabilities during the raster scanning process [21]. These three techniques (X-PEEM, MTXM and STXM) have matured since and dominate the field of magnetic X-ray imaging. X-PEEM and MTXM are full-field microscopes, which allow taking images covering a large field of view images within a few seconds of exposure time. STXM has the advantage in employing various point detection schemes, including fast photodetectors, which can pick individual bunches from the x-ray source [22]. They can also record the local fluorescence yield or the sample current or can be even combined with a scanning probe microscope, such as an AFM [23]. The inherent downside of STXM is that the image acquisition time for a field of view of several micrometer is about 100 times slower than with the full-field x-ray microscopes at similar spatial resolutions [24], which clearly favors X-PEEM and TXM for multidimensional studies.

Besides the conventional microscopy approach, i.e., using optical elements to build a microscope, the x-ray community is exploring microscopy techniques, which allow retrieving microscopic images solely from the diffraction of the $\mathrm{x}$-rays in the sample. These techniques are broadly called lensless $\mathrm{x}$-ray microscopies. They rely on the coherence properties of the $\mathrm{x}$-rays, which in reverse drive the development of next generation $\mathrm{x}$-ray sources, such as X-ray Free Electron Lasers or diffraction limited storage rings (DLSR)offering nearly fully coherence both longitudinal and transversal. To this class of $\mathrm{x}$-ray microscopies belongs $\mathrm{x}$ ray holography [25], where a reference hole next to the sample provides a reference beam and a Fast Fourier transform is performed to obtain a real space image. In Coherent Diffractive Imaging (CDI) a phase retrieval algorithm is engaged [26] and in X-ray ptychography diffraction patterns recorded with a STXM for every step in the scanning process are used to retrieve a real space image at higher spatial resolution, than what the FZP used in the STXM for this experiment can provide [27]. As the diffraction more or less inherently is an interference experiment, these CDI approaches give simultaneous access to both amplitude and phase contrast, which in case of real space microscopes requires additional optics or other detection schemes. Whereas in cases, where the absorption is rather weak, e.g., in soft tissues, a phase contrast can enhance the information contained in the image, the similar size of magnetic absorption (XMCD) and magnetic phase contrast does not offer substantial advantages for magnetic phase contrast [28]. In principle, as absorption and phase are interrelated by Kramers-Kronig transformations [29], the quantitative information (magneto-optical sum rules) contained in the $\mathrm{x}$-ray absorption channel should be obtainable from the $\mathrm{x}$-ray phase channel as well. 


\section{WHAT HAS BEEN ACHIEVED SO FAR WITH MAGNETIC X-RAY MICROSCOPIES AND WHAT ARE THE MAJOR CHALLENGES THAT LIE AHEAD?}

Probably the parameter, which is most directly associated with any type of microscopy and where its capability is judged against, is the spatial resolution. For optical microscopies the diffraction limit, i.e., according to Abbe half of the wavelength, which is typically around $250 \mathrm{~nm}$ can be overcome to a certain degree by super-resolution concepts and has recently led to the 2014 Nobel Prize for Chemistry [30]. X-ray microscopies are still far away from an analogous limit. Albeit state-of-the-art Fresnel zone plate optics have demonstrated $10 \mathrm{~nm}$ resolution [31], [32], XPEEM with aberration correction is approaching similar length scales and ptychography results have recently achieved even a sub-10 nm resolution with (non-magnetic) test patterns [33], the day-to-day imaging of magnetic structures with $\mathrm{x}$-ray microscopies is around $20 \mathrm{~nm}$ spatial resolution. In this context, it is noteworthy, that coherent scattering with soft $\mathrm{x}$-rays is capable to determine correlation lengths at the wavelength and provides detailed information of the Fourier components of the ordering. Hence, it allows retrieving significant structural and magnetic information in the $\mathrm{nm}$ regime complementing the "real imaging" techniques, described here. Pushing the spatial resolution with $\mathrm{x}$-ray microscopies is at the forefront of $\mathrm{x}$-ray research but it remains to be seen, when and if a spatial resolution at the ultimate diffraction limit for $\mathrm{x}$-rays can be achieved in experiments, which go beyond test patterns with a well defined and a-priori known structure and a high contrast.

The second appealing feature of magnetic $\mathrm{x}$-ray microscopes is the capability to image with high spatial resolution dynamical processes. For magnetic materials, the relevant time scales range from the ultimate time scales of the exchange interaction in the fsec regime across the psec regime, where spin-orbit phenomena dominate, up to the nsec time scale, where precession and domain wall motion occurs. Over the last few years, time-resolved $\mathrm{x}$-ray microscopies at $3 \mathrm{rd}$ generation synchrotrons have made substantial contributions e.g., to deepen our understanding of the gyrational dynamics in magnetic vortex structures. Magnetic vortices form, e.g., ferromagnetic micron sized disks and their spin structure resembles the energetic ground state. It consists of an in-plane circulating component following the circumference of the disk and a vortex core at the center, where the magnetization points orthogonal to the disk plane [34]. The major limitation in time-resolved $\mathrm{x}$-ray microscopy experiments is that the low intensity per single $\mathrm{x}$-ray pulse requires a stroboscopic pumpprobe scheme, which restricts any time-resolved $\mathrm{x}$-ray microscopy on the sub-nsec regime to fully reproducible processes. Vortex gyration, which can be induced by magnetic or current field pulses in confined magnetic elements, such as circles, squares, ellipses and rectangles are perfectly repeatable and suited for that. Controlling both the polarity, i.e., the vortex core orientation [35] and the circularity, i.e., the sense of rotation of the in-plane spin configuration [36] was demonstrated, although details in these processes, which occur on much faster time scales, are still experimentally inaccessible with current $\mathrm{x}$-ray microscopies. The dynamics of stochastic or non-deterministic processes, which are the more general case for spin dynamics is still not accessible either. The high peak brightness at currently available XFELs should be sufficient to perform single-shot experiments [37] and, with microscopic spatial information, however, in most cases, there will be severe sample damage (even destroyed) already after the first shot. To study the temporal evolution on fast time scales with these destructive probes will therefore require a fully reproducible sample quality for each of the single-shots, which will be very challenging to achieve in most cases. Even more important seems to be the requirement to follow the dynamics over multiple time scales when different interaction set in that can control the final outcome of the steady state.

The most important capability of using polarized $\mathrm{x}$-rays for the study of magnetic materials is the ability to quantify with elemental specificity their magnetic properties, specifically to distinguish between spin and orbital magnetic moments. As magnetic X-ray microscopies utilize those magnetic dichroism effects as magnetic contrast mechanism, any $\mathrm{x}$-ray microscope is inherently capable of retrieving information on spin and orbital magnetic moments locally with high spatial resolution. This opens the door to test the design of novel magnetic materials, e.g., for bit patterned media, which rely on a local control of magnetic anisotropies. Examples demonstrating these feasibilities are X-PEEM studies of the spin reorientation transition in a wedge shaped thin Ni film [38] or a recent MTXM experiment, which showed a change of L/S ratio at the domain wall in a magnetic film with perpendicular anisotropy [39].

Time resolved XMCD experiments using e.g., the femtoslicing source at the BESSY II synchrotron in Berlin harness this quantitative feature of XMCD spectroscopy and have revealed the temporal evolution of spin and orbital magnetic moments on fsec time scales [40]. Yet, the combination with spatial resolution, i.e., its local character remains elusive so far due to the limited photon flux at a slicing source.

Moving into multidimensional characterization is clearly one of the major frontiers for magnetic $\mathrm{x}$-ray microscopies. One direction is to go beyond two dimensional imaging of magnetic spin structures and take into account the three dimensional arrangement of spins. This tackles the spatiotemporal characterization of buried interfaces in magnetic multilayered structures or superlattices, the polarization of non-magnetic materials in proximity to magnetic materials, more generally, the ability to trace the depth profile of magnetization in layered magnetic structures or to investigate magnetic behavior, specifically the magnetization reversal in 3dim systems, such as nanowires, where Bloch points seem to play an important role. Other examples are magnetic behavior of core-shell nanoparticles or the spin configuration in magnetic hollow spheres. Magnetic tomography at high spatial resolution is currently also explored with other probes, e.g., with Lorentz-TEM [41]. However, magnetic tomography with x-ray microscopes will enable not only to achieve structural information, but add quantitative information and maybe reveal the spin dynamics in 3dim as well. First steps into this direction are rolledup magnetic thin films [42], which can be fabricated by strain engineering and exhibit an increased magneto-resistance which scales with the winding number of the films [43]. Such systems can be seen as prototypes for a shapeable spintronics in analogy to shapeable electronics [44]. 
The other challenge for magnetic $\mathrm{x}$-ray microscopies is the desire to increase its sensitivity, which can be accomplished with higher photon flux, i.e., brighter sources or with improved detection schemes. This will be of paramount importance to investigate systems with single or a few spins only, e.g., in molecular magnets or diluted magnetic semiconductors. Finally, the detection of pure spin currents or spin accumulation in lateral spin valves would benefit tremendously from an increased sensitivity.

Although magnetic imaging with polarized $\mathrm{x}$-rays is a rather young scientific discipline, the various types of established $\mathrm{x}$-ray microscopes have already taken an important role in state-ofthe-art characterization of the properties and behavior of spin textures in advanced materials. The opportunities ahead will be to obtain in a unique way indispensable multidimensional information of the structure, dynamics and composition of scientifically interesting and technologically relevant magnetic materials.

New sources and facilities are on the horizon, which will facilitate harnessing the full potential of the interaction of polarized soft $\mathrm{x}$-rays with magnetic materials, which will be made visible in magnetic $\mathrm{x}$-ray microscopies.

This work was supported by the Director, Office of Science, Office of Basic Energy Sciences, Materials Sciences and Engineering Division, of the U.S. Department of Energy under Contract No. DE-AC02-05-CH11231 and by the Leading Foreign Research Institute Recruitment Program (Grant No. 2012K1A4A3053565) through the National Research Foundation of Korea (NRF) funded by the Ministry of Education, Science and Technology (MEST).

\section{REFERENCES}

1. Bader SD, Parkin SSP. Spintronics. Annu Rev Condens Matter Phys. (2010) 1:71. doi: 10.1146/annurev-conmatphys-070909-104123

2. Gutfleisch O, Willard MA, Brück E, Chen CH, Sankar SG, Ping Liu J. Magnetic materials and devices for the 21st century: stronger, lighter, and more energy efficient. Adv Mater. (2010) 23:821. doi: 10.1002/adma.201002180

3. Emori S, Bauer U, Ahn S-M, Martinez E, Beach GSD. Current-driven dynamics of chiral ferromagnetic domain walls. Nat Mater. (2013) 12:611. doi: $10.1038 /$ nmat3675

4. Weller D, Mosendz O, Parker G, Pisana S, Santos TS. L10 FePtX-Y media for heat-assisted magnetic recording. Phys Stat Sol. (2013) 210:1245. doi: 10.1002/pssa.201329106

5. Beaurepaire E, Merle J-C, Daunois A, Bigot J-Y. Ultrafast spin dynamics in ferromagnetic nickel. Phys Rev Lett. (1996) 76:4250. doi: 10.1103/PhysRevLett. 76.4250

6. Kirilyuk A, Kimel AV, Rasing T. Laser-induced magnetization dynamics and reversal in ferrimagnetic alloys. Rep Prog Phys. (2013) 76:026501. doi: 10.1088/0034-4885/76/2/026501

7. Lambert C-H, Mangin S, Varaprasad BS, Takahashi YK, Hehn M, Cinchetti $\mathrm{M}$, et al. All-optical control of ferromagnetic thin films and nanostructures. Science (2014) 345:1337. doi: 10.1126/science. 1253493

8. Hubert A, Schäfer R. Magnetic Domains: The Analysis of Magnetic Microstructure. Berlin: Springer-Verlag (1998).

9. Kuch W, Schäfer R, Fischer P, Hillebrecht U. Magnetic Microscopy of Layered Structures. In: Springer Series in Surface Sciences Vol. 57. Berlin; Heidelberg: Springer Verlag (2015).

10. van der Laan G. Applications of soft x-ray magnetic dichroism. J Phys Conf Ser. (2013) 430:012127. doi: 10.1088/1742-6596/430/1/012127

11. Radu I, Stamm C, Eschenlohr A, Radu F, Abrudan R, Vahaplar K, et al. Engineering ultrafast magnetism in ultrafast magnetism I. In: Springer Proceedings in Physics Vol. 159. Strasbourg (2015). p. 297.

12. Silva TJ, Turgut E, Mathias S, La-o-vorakiat C, Grychtol P, Adam R, et al. Ultrafast, element-specific magnetization dynamics of multi-constituent magnetic materials by use of high-harmonic generation. In: Ultrafast Magnetism
I. Springer Proceedings in Physics Vol. 159. Strasbourg (2015). p. 300. doi: 10.1007/978-3-319-07743-7_93

13. Eriksson M, van der Veen JF, Quitmann C. Diffraction-limited storage rings - a window to the science of tomorrow. J Synchr Rad. (2014) 21:837. doi: 10.1107/S1600577514019286

14. Peele AG, Nugent KA, Mancuso AP, PatersonD, McNulty I, Hayes JP. X-ray phase vortices: theory and experiment. J Opt Soc Am A Opt Image Sci Vis. (2004) 21:1575. doi: 10.1364/JOSAA.21.001575

15. Chen CT, Sette F, Ma Y, Modesti S. Soft X-ray magnetic circular dichroism at the $\mathrm{L}_{2,3}$ edges of nickel. Phys Rev B Condens Matter (1990) 42:7262. doi: 10.1103/PhysRevB.42.7262

16. Thole BT, Carra P, Sette F, van der Laan G. X-ray circular dichroism as a probe of orbital magnetization. Phys Rev Lett. (1992) 68:1943. doi: 10.1103/PhysRevLett.68.1943

17. Carra P, Thole BT, Altarelli M, Wang X. X-ray circular dichroism and local magnetic fields. Phys Rev Lett. (1993) 70:694. doi: 10.1103/PhysRevLett.70.694

18. Chen CT, Idzerda YU, Lin H-J, Smith NV, Meigs G, Chaban E, et al. Experimental confirmation of the X-ray magnetic circular dichroism sum rules for iron and cobalt. Phys Rev Lett. (1995) 75:152. doi: 10.1103/PhysRevLett. 75.152

19. Stohr J, Wu Y, Hermsmeier BD, Samant MG, Harp G, Koranda S, et al. Element-specific magnetic microscopy with circularly polarized X-rays. Science (1993) 259:5095.

20. Fischer P, Schutz G, Schmahl G, Guttmann P, Raasch D. Imaging of magnetic domains with the X-ray microscope at BESSY using X-ray magnetic circular dichroism. Zeitschrift für Physik B Condens Matter (1996) 101:313. doi: 10.1007/s002570050214

21. Kilcoyne AL, Tyliszczak T, Steele WF, Fakra S, Hitchcock P, Franck K, et al. Interferometer-controlled scanning transmission X-raymicroscopes at the advanced light source. J Synchr Rad. (2003) 10:125. doi: 10.1107/S0909049502017739

22. Acremann Y, Strachan JP, Chembrolu V, Andrews SD, Tyliszczak T, Katine JA, et al. Time-resolved imaging of spin transfer switching: beyond the macrospin concept. Phys Rev Lett. (2006) 96:217202. doi: 10.1103/PhysRevLett.96. 217202

23. Pilet N, Raabe J, Stevenson SE, Romer S, Bernard L, McNeill CR, et al. Nanostructure characterization by a combined x-ray absorption/scanning force microscopy system. Nanotechnology (2012) 23:475708. doi: 10.1088/0957-4484/23/47/475708

24. Guttmann P, Bittencourt C, Rehbein S, Umek P, Ke X, Van Tendeloo G, et al. Nanoscale spectroscopy with polarized X-rays by NEXAFS-TXM. Nat Photonics (2012) 6:25. doi: 10.1038/nphoton.2011.268

25. Eisebitt S, Luening J, Schlotter WF, Loergen M, Hellwig O, Eberhardt W, et al. Lensless imaging of magnetic nanostructures by X-ray spectro-holography. Nature (2004) 432:885. doi: 10.1038/nature03139

26. Tripathi A, Mohanty J, Dietze SH, Shpyrko OG, Shipton E, Fullerton EE, et al. Dichroic coherent diffractive imaging. Proc Natl Acad Sci USA. (2011) 108:13393. doi: 10.1073/pnas.1104304108

27. Dierolf M, Menzel A, Thibault P, Schneider P, Kewish CM, Wepf R, et al. Ptychographic X-ray computed tomography at the nanoscale. Nature (2010) 467:436. doi: 10.1038/nature09419

28. Chang C, Sakdinawat A, Fischer P, Anderson E, Attwood D. Single-element objective lens for soft x-ray differential interference contrast microscopy. Opt Lett. (2006) 31:1564. doi: 10.1364/OL.31.001564

29. Kortright JB, Kim SK. Resonant magneto-optical properties of $\{\mathrm{Fe}\}$ near its 2p levels: Measurement and applications. Phys Rev B (2000) 62:12216. doi: 10.1103/PhysRevB.62.12216

30. Klar TA, Jakobs S, Dyba M, Egner A, Hell SW. Fluorescence microscopy with diffraction resolution barrier broken by stimulated emission. Proc Natl Acad Sci USA. (2000) 97:8206. doi: 10.1073/pnas.97.15.8206

31. Chao W, Kim J, Rekawa S, Fischer P, Anderson EH. Real space soft xray imaging at $10 \mathrm{~nm}$ spatial resolution. Opt Expr. (2012) 20:9777. doi: 10.1364/OE.20.009777

32. Vila-Comamala J, Jefimovs K, Raabe J, Fink RH, Senoner M, Maszdorf A, et al. Advanced thin film technology for ultra high resolution X-ray microscopy. Ultramicroscopy (2009) 109:1360. doi: 10.1016/j.ultramic.2009.07.005

33. Shapiro D, Yu Y-S, Tyliszczak T, Cabana J, Celestre R, Chao W, et al. Chemical composition mapping with nanometre resolution by soft X-ray microscopy. Nat Photonics (2014) 8:765. doi: 10.1038/nphoton.2014.207 
34. Shinjo T, Okuno T, Hassdorf R, Shigeto K, Ono T. Magnetic vortex core observation in circular dots of permalloy. Science (2000) 289:930. doi: 10.1126/science.289.5481.930

35. Van Waeyenberge B, Puzic A, Stoll H, Chou KW, Tyliszczak T, Hertel R, et al. Magnetic vortex core reversal by excitation with short bursts of an alternating field. Nature (2006) 444:461. doi: 10.1038/nature05240

36. Uhlir V, Urbanek M, Hladik L, Spousta J, Im M-Y, Fischer P, et al. Dynamic switching of the spin circulation in tapered magnetic nanodisks. Nat Nanotechnol. (2013) 8:341. doi: 10.1038/nnano.2013.66

37. Wang T, Zhu D, Wu B, Graves C, Schaffert S, Rander T, et al. Femtosecond single-shot imaging of nanoscale ferromagnetic order in $\mathrm{Co} / \mathrm{Pd}$ multilayers using resonant x-ray holography. Phys Rev Lett. (2012) 108:267403. doi: 10.1103/PhysRevLett.108.267403

38. Kuch W, Gilles J, Kang S, Imada S, Suga S, Kirschner J. Magnetic-circulardichroism microspectroscopy at the spin reorientation transition in $\mathrm{Ni}(001)$ films. Phys Rev B (2000) 62:3824. doi: 10.1103/PhysRevB.62.3824

39. Robertson MJ, Agostino CJ, N'Diaye AT, Chen G, Im M-Y, Fischer P. Quantitative XMCD mapping with high spatial resolution full-field magnetic transmission soft x-ray spectro-microscopy. J Appl Phys. (2014). (in press).

40. Radu I, Vahaplar K, Stamm C, Kachel T, Pontius N, Duerr HA, et al. Transient ferromagnetic-like state mediating ultrafast reversal of antiferromagnetically coupled spins. Nature (2011) 472:205. doi: 10.1038/nature 09901

41. Phatak C, Liu Y, Gulsoy EB, Schmidt D, Franke-Schubert E, PetfordLong A. Visualization of the magnetic structure of sculpted threedimensional cobalt nanospirals. Nano Lett. (2014) 14:759. doi: 10.1021/ nl404071u
42. Streubel R, Makarov D, Karnaushenko D, Han L, Schmidt OG, Kim S-K, et al. Magnetic microstructure of rolled-up single-layer ferromagnetic nanomembranes. Adv Mater. (2014) 26:316. doi: 10.1002/adma.201303003

43. Mueller C, Bof Bufon CC, Makarov D, Fernandez-Outon LE, Waldemar AA, Schmidt OG, et al. Tuning giant magnetoresistance in rolled-up Co$\mathrm{Cu}$ nanomembranes by strain engineering. Nanoscale (2012) 4:7155. doi: $10.1039 / \mathrm{c} 2 \mathrm{nr} 32086 \mathrm{j}$

44. Melzer M, Makarov D, Calvimontes A, Karnaushenko D, Baunack S, Kaltofen $\mathrm{R}$, et al. Stretchable magnetoelectronics. Nano Lett. (2011) 11:2522. doi: $10.1021 / \mathrm{nl} 201108 \mathrm{~b}$

Conflict of Interest Statement: The author declares that the research was conducted in the absence of any commercial or financial relationships that could be construed as a potential conflict of interest.

Received: 25 October 2014; accepted: 08 December 2014; published online: 08 January 2015.

Citation: Fischer $P$ (2015) Frontiers in imaging magnetism with polarized $x$-rays. Front. Phys. 2:82. doi: 10.3389/fphy.2014.00082

This article was submitted to Condensed Matter Physics, a section of the journal Frontiers in Physics.

Copyright (c) 2015 Fischer. This is an open-access article distributed under the terms of the Creative Commons Attribution License (CC BY). The use, distribution or reproduction in other forums is permitted, provided the original author(s) or licensor are credited and that the original publication in this journal is cited, in accordance with accepted academic practice. No use, distribution or reproduction is permitted which does not comply with these terms. 